\title{
Original article (short paper) \\ Influence of physical activity and different sensory conditions on static and dynamic balance of pregnant women
}

\author{
Vanessa Patrícia Soares de Sousa \\ Aline da Silva Santos \\ Ana Paula Spaniol \\ Elizabel de Souza Ramalho Viana \\ Universidade Federal do Rio Grande do Norte, Natal, RN, Brasil
}

\begin{abstract}
Background: Physical activity during pregnancy provides countless benefits to pregnant women and results in a smaller number of falls during pregnancy. Thus, this study aims to verify the influence of physical activity and test conditions on the static and dynamic balance of pregnant women. Methodology: The study was an analytical, transversal, and comparative study with a sample of 99 pregnant women divided into two groups, with physical activity as the grouping factor. The evaluation included questions about the type and frequency of physical activity and investigation of postural balance (Balance Master System $\left.{ }^{\circledR}\right)$. For statistical analysis, we used the following tests: Shapiro Wilk, Mann-Whitney, ANOVA One Way and Wilcoxon. Results: No relationship was found between physical activity and postural balance $(\mathrm{F}>0.40, \mathrm{P}>0.07)$. However, the test conditions alter the sway velocity $(\mathrm{P}=0.001)$ and directional control movement $(\mathrm{P}=0.001)$. Conclusions: Results suggest that: (1) physical activity does not influence variables related to postural balance in active and sedentary pregnant women; and (2) postural sway velocity and directional control of movement are related to the test conditions used during balance evaluation.
\end{abstract}

Keywords: postural balance, exercise, pregnancy

\section{Introduction}

Postural balance is the ability to maintain one's center of gravity within one's support base during either internal or external destabilization ${ }^{1}$. Pregnant women are more prone to fall due to the physiological and biomechanical changes that occur during pregnancy, which reduce postural balance ${ }^{1-6}$. Findings in the literature have suggested that posture stability decreases with the advance of gestation. McCory and colleagues evaluated the effect of exercise on the risk of falls in pregnant women. They found that in active pregnant women, the risk of falls is lower?

Given that the literature has reported the benefits of proper practice exercise on this body function ${ }^{8,9}$, sedentary lifestyle and insufficient physical activity can negatively affect processes related to postural balance control. This association is justified by the fact that physical activity during pregnancy helps maintain muscle strength at adequate levels, which is important for proper maintenance of postural balance ${ }^{10}$.

Given these facts, this study aims to analyze the influence of physical activity on the variables of the dynamic and static postural balance in active healthy pregnant women and sedentary ones. Furthermore, the influence of visual afference, surface type, and direction of movement were observed for static and dynamic postural balance.

\section{Methodology}

\section{Study Design}

This study was characterized as analytical and transverse. It was approved by the Ethics Committee on Human Research of the
Federal University of Rio Grande do Norte (protocol number: 719.939), and all volunteers signed the consent form.

\section{Environment and Period of Research}

The research was conducted in the Department of Physiotherapy of the Federal University of Rio Grande do Norte, in the Laboratory of Neuromuscular Performance Analysis (LAPERN). The study period was August 2013 to October 2014.

\section{Participants}

A sample of 99 pregnant women were recruited through convenience sampling from a population of 250 pregnant women assisting in the Preparatory Course to Pregnancy-Labour and Postpartum. For the initial analysis, the sample was divided into two groups with physical activity (yes or no) as the grouping factor. Information on physical activity was collected through structured interviews with the following questions:

1. Do you engage regularly in physical activity? Response options: yes or no

2. How many times a week do you engage in physical activity?

3. What physical activities do you engage in?

Two groups were formed: active $(n=46)$ and sedentary $(n=53)$. Later, these two groups were divided by trimester of pregnancy: active women in the second trimester $(2 \mathrm{TA} ; \mathrm{n}=19)$, sedentary women in the second trimester $(2 \mathrm{TS} ; \mathrm{n}=25)$, active women in the third trimester (3TA; $\mathrm{n}=27)$, and sedentary women in the third trimester (3TS; $\mathrm{n}=28$ ). 


\section{Data Acquisition and Evaluation Procedures}

Clinical information, obstetric information, and changes related to pregnancy were collected through an instrument designed by the lead researchers. After this initial data collection, the subjects were questioned about their physical activity and the type and weekly frequency of their activity, if any.

For assessment of balance, the Balance Master System ${ }^{\circledR}$ (NeuroCom, Clackamas, USA) was used. This is a system with a force plate with four transducers, which measure the vertical reaction forces exerted by an individual's feet. Based on data from the center of pressure (COP) and the subject's height, an estimate of the center of gravity $(\mathrm{CG})$ is calculated. Static and dynamic postural balance were analyzed with the Modified Clinical Test of Sensory Interaction on Balance (mCTSIB) and Rhythmic Weight Shift Test (RWS). The validity of these tests was confirmed in work conducted by Liston and Brouwer ${ }^{11}$.

During the execution of the mCTSIB test, participants had their velocity of postural sway evaluated in four different situations, obtaining three measurements for each test condition: (1) firm surface and eyes open (FEO), (2) firm surface and eyes closed (FEC), (3) unstable surface and eyes open (UEO), and (4) unstable surface and eyes closed (UEC). Each test attempt lasted 10 seconds (time set by device). In all cases, the volunteer was instructed to keep the upper limbs near and along the body.

The Rhythmic Weight Shift Test (RWS) resembles a virtual reality game in which the subject is represented on the computer screen by an avatar. Before starting the test, participants were instructed to remain with their arms beside their bodies and the torso upright and straight. They were instructed to do side-toside and anterior-posterior weight shifts at three different speeds (low, medium, and high), which caused the avatar to appear with two parallel bars displayed on the computer screen during the test. Afterwards, the following variables related to dynamic postural balance were analyzed: oscillation velocity during lateral-lateral displacement (VELOC_LL) and anterior-posterior (VELOC_AP) of weigh; directional control in lateral-lateral direction (CD_LL) and anterior posterior (CD_AP).

The support base was defined by markings on the force platform. The subjects' feet were positioned by aligning the lateral border of each foot with the appropriate height line marked on the force plates (i.e., short, $76-140 \mathrm{~cm}$; medium, $141-165 \mathrm{~cm}$; or tail, 166-203cm). The medial malleolus was aligned with the transverse force plate line, and subjects adopted a comfortable amount of forefoot splay. The visual display monitor was adjusted to the subject's eye level ${ }^{11}$.

\section{Statistical Analysis}

To verify the normality of quantitative variables, the ShapiroWilk test was used. Sample characterization in terms of sociodemographic and obstetric data were presented as mean and standard deviation, absolute and relative frequencies, depending on the type of variable (quantitative or categorical). To identify the types and weekly frequency of physical activity among active pregnant women, descriptive statistics and the data were presented on relative and absolute frequencies. The Mann-Whitney test was used to compare variables of postural balance between the groups (active and sedentary). When stratifying the sample into four groups by gestational trimesters and physical activity, a one-way ANOVA was applied to analyze the behavior of the postural balance variables among the groups. Finally, the Wilcoxon test was used to evaluate the influence of test conditions (visual afferency, type of surface, and direction of the weight shift) on oscillation speed and postural control for the sample as a whole. A statistical significance of $5 \%$ was adopted.

\section{Sample Calculation and Bias Control}

To obtain the data needed to calculate the sample, a pilot study was conducted with 30 volunteers ( 15 active and 15 sedentary). Through this pre-experiment, we obtained an average of $0.26 \%$ and a standard deviation of $0.12 \%$, with the speed of postural sway as the main variable of this study. These values were added to a virtual calculator (http://www.lee.dante.br), adopting a power test as $80 \%$ and a significance level of $5 \%$, and a sample size of 92 volunteers as valid for conducting the study (46 active and 46 sedentary). To avoid possible bias in the acquisition and interpretation of data, the following procedures were adopted: (1) assessors were trained in the use of the questionnaire and the machine to evaluate postural balance; and (2) control of the main confounding variables (gestational age, height, and weight), with respect to postural balance.

\section{Results}

The mean chronological and gestational ages were, respectively, $29.9 \pm 4.9$ years and $25.4 \pm 5.4$ weeks. We found that $46.5 \%$ of the sample practiced physical activity. Further analyses are shown in Table 1.

Table 1- Sociodemographic, clinical, obstetrical, anthropometric, and lifestyle characteristics of the total sample $(n=99)$.

\begin{tabular}{lccc}
\hline \multicolumn{1}{c}{ Variables } & Total $(\mathbf{n}=\mathbf{9 9 )}$ & $\mathbf{2 T}(\mathbf{n}=\mathbf{4 4})$ & $\mathbf{3 T}(\mathbf{n}=\mathbf{5 5})$ \\
\hline $\begin{array}{l}\text { Data Sociodemographic } \\
\text { Age (in years) }\end{array}$ & $29,9 \pm 4,8$ & $30,0 \pm 5,9$ & $29,9 \pm 3,7$ \\
Education & & & $1,8 \%(1)$ \\
High School & $5,1 \%(5)$ & $9,1 \%(4)$ & $98,2 \%(54)$ \\
Higher Education & $94,9 \%(94)$ & $90,4 \%(40)$ & $3,6 \%(2)$ \\
Family income & $4 \%(4)$ & $4,5 \%(2)$ & \\
1 a 2 MW & & & \\
\hline
\end{tabular}




\begin{tabular}{|c|c|c|c|}
\hline Variables & Total $(n=99)$ & $2 T(n=44)$ & $3 T(n=55)$ \\
\hline 3 a $4 \mathrm{MW}$ & $16,2 \%(16)$ & $13,6 \%(6)$ & $18,2 \%(10)$ \\
\hline$>4 \mathrm{MW}$ & $68,7 \%(8)$ & $65,9 \%(29)$ & $70,9 \%(39)$ \\
\hline Do not answer & $11,1 \%(11)$ & $15,9 \%(7)$ & $7,3 \%(4)$ \\
\hline \multicolumn{4}{|l|}{ Data Obstetric } \\
\hline GA (in weeks) & $25,4 \pm 5,4$ & $20,3 \pm 3,3$ & $29,5 \pm 2,5$ \\
\hline \multicolumn{4}{|c|}{ Data anthropometric and lifestyle } \\
\hline Weight (in $\mathrm{Kg}$ ) & $67,6 \pm 11,9$ & $66,5 \pm 8,7$ & $68,5 \pm 1,4$ \\
\hline Height (in meters) & $1,61 \pm 0,06$ & $1,61 \pm 0,06$ & $1,62 \pm 0,06$ \\
\hline \multicolumn{4}{|l|}{ Physical activity } \\
\hline Yes & $46,5 \%(46)$ & $43,2 \%(19)$ & $49,1 \%(27)$ \\
\hline No & $53,5 \%(53)$ & $56,8 \%(25)$ & $50,9 \%(28)$ \\
\hline
\end{tabular}

NOTE: Categorical variables are presented in relative frequency (absolute frequency). The numerical variables are presented as mean and standard deviation. LEGEND: MW, minimum wage (R\$ 724,00); AG, Gestational age; 2T-Second trimester; 3T-Third trimester.

The physical activities most practiced by the active volunteers $(n=46)$ were Pilates $(26.1 \%)$ and water aerobics $(13 \%)$.
The most commonly reported frequency was 2 times a week (Table 2).

Table 2-Type and frequency of physical activities practiced by active pregnant women $(n=46)$

\begin{tabular}{|c|c|c|c|}
\hline Variables & Active pregnant $(n=46)$ & $2 T(n=19)$ & $3 T(n=27)$ \\
\hline \multicolumn{4}{|l|}{ Physical activity } \\
\hline Pilates & $26,1 \%(12)$ & $26,3 \%(5)$ & $25,9 \%(7)$ \\
\hline Water aerobics & $13 \%(5)$ & $15,9 \%(3)$ & $11,1 \%(3)$ \\
\hline Walking & $13 \%(5)$ & $10,5 \%(2)$ & $14,8 \%(4)$ \\
\hline Dance & $2,2 \%(1)$ & $5,3 \%(1)$ & - \\
\hline Yoga & $2,2 \%(1)$ & - & $3,7 \%(1)$ \\
\hline Weight lifting & $6,5 \%(3)$ & - & $11,1 \%(3)$ \\
\hline Bicycle & $2,2 \%(1)$ & - & $3,7 \%(1)$ \\
\hline Swimming & $2,2 \%(1)$ & - & $3,7 \%(1)$ \\
\hline Pilates and Walking & $6,5 \%(3)$ & $10,5 \%(2)$ & $3,7 \%(1)$ \\
\hline Pilates and Water aerobics & $6,5 \%(3)$ & - & $11,1 \%(3)$ \\
\hline Walking and Yoga & $4,3 \%(2)$ & $10,5 \%(2)$ & - \\
\hline Walking and Dance & $2,2 \%(1)$ & $5,3 \%(1)$ & - \\
\hline Walking and Weight lifting & $2,2 \%(1)$ & - & $3,7 \%(1)$ \\
\hline Water aerobcs and Walking & $2,2 \%(1)$ & - & $3,7 \%(1)$ \\
\hline Water aerobcs and Yoga & $2,2 \%(1)$ & $5,3 \%(1)$ & \\
\hline Not reported & $6,5 \%(3)$ & $10,5 \%(2)$ & $3,7 \%(1)$ \\
\hline \multicolumn{4}{|l|}{ Frequency } \\
\hline $1 \mathrm{x}$ per week & $2,2 \%(1)$ & $5,3 \%(1)$ & - \\
\hline $2 \mathrm{x}$ per week & $41,3 \%(19)$ & $36,8 \%(7)$ & $44,4 \%(12)$ \\
\hline $3 x$ per week & $37 \%(17)$ & $42,1 \%(8)$ & $33,3 \%(9)$ \\
\hline $4 \mathrm{x}$ per week & $6,5 \%(3)$ & $5,3 \%(1)$ & $7,4 \%(2)$ \\
\hline $5 x$ per week & $6,5 \%(3)$ & - & $11,1 \%(3)$ \\
\hline Not reported & $6,5 \%(3)$ & $10,5 \%(2)$ & $3,7 \%(1)$ \\
\hline
\end{tabular}

NOTE: The variables are presented in relative frequency (absolute frequency). LEGEND: 2T, second trimester; 3T, third trimester.

Sedentary women were observed to show greater postural sway velocity than their active counterparts $(P=0.03)$ during the static balance task on the firm surface a and open eyes condition (Figure 1). No other variables for static or dynamic balance showed any significant differences $(\mathrm{P}>0.17)$.

Considering the gestational trimesters (second $\mathrm{x}$ third) and physical activity (active $\times$ sedentary), no statistically significant differences were observed between groups $(\mathrm{F}>0.40, \mathrm{P}>0.07)$ (Table 3).
The analysis of the influence of test conditions on the volunteers' postural balance variables showed greater sway velocity $(\mathrm{SV})$ on unstable surfaces $(\mathrm{P}<0.001)$ and with closed eyes $(\mathrm{P}<0.001)$, during the static balance test (Figure 2).

The measurement of dynamic balance showed a higher SV during side-to-side movement $(\mathrm{P}<0.001)$ (Figure $3 \mathrm{~A})$ and lower directional control while moving in the anteroposterior direction $(\mathrm{P}<0.001)$ (Figure 3B). 


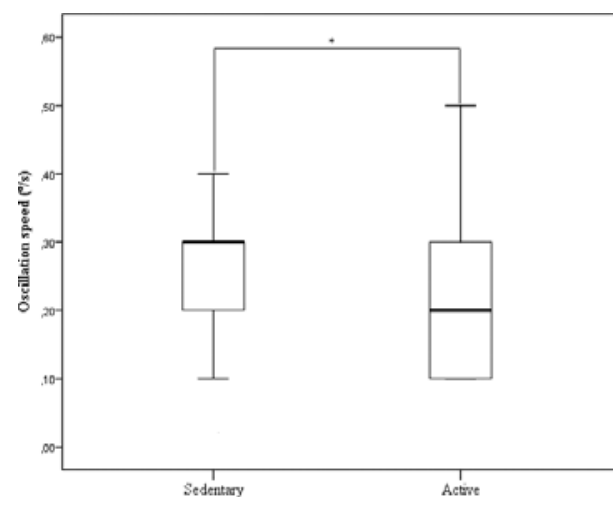

Figure 1 - Analysis of postural sway velocity on the firm surface and open eyes, when comparing the active and sedentary women. $* \mathrm{P}<0.05$

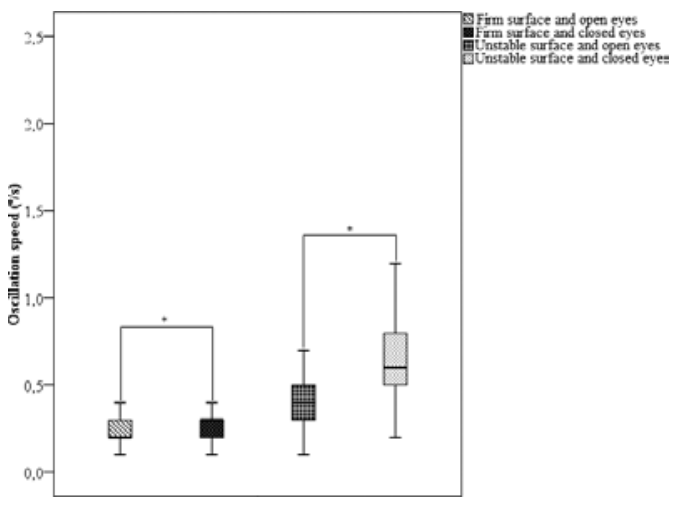

Figure 2 - Analysis of postural sway velocity in different sensory conditions (with or without visual feedback) and surfaces (firm or unstable). $* \mathrm{P}<005$

Table 3- Behavior analysis of the variables of postural balance, with gestational trimesters and physical activity as grouping factors.

\begin{tabular}{|c|c|c|c|c|c|c|}
\hline \multirow[t]{2}{*}{ Variables } & \multicolumn{3}{|c|}{ Groups } & \multicolumn{3}{|c|}{ IC $95 \%$} \\
\hline & 2TA $(n=19)$ & 2TS $(n=25)$ & 3TA $(n=27)$ & $3 T S(n=28)$ & Lower bound Upper bound & $\mathbf{P}$ \\
\hline \multicolumn{7}{|l|}{ Static Balance } \\
\hline FEO $(\mathrm{em} \%$ s) & $0,22 \pm 0,11$ & $0,28 \pm 0,11$ & $0,21 \pm 0,10$ & $0,24 \pm 0,09$ & $0,17-0,25$ & 0,15 \\
\hline $\mathrm{FEC}(\mathrm{em} \% \mathrm{~s})$ & $0,23 \pm 0,11$ & $0,29 \pm 0,13$ & $0,24 \pm 0,12$ & $0,26 \pm 0,15$ & $0,17-0,28$ & 0,41 \\
\hline UEO $(\mathrm{em} \% \mathrm{~s})$ & $0,35 \pm 0,11$ & $0,40 \pm 0,17$ & $0,38 \pm 0,13$ & $0,38 \pm 0,11$ & $0,30-0,41$ & 0,74 \\
\hline $\operatorname{UEC}(\mathrm{em} \% \mathrm{~s})$ & $0,64 \pm 0,20$ & $0,63 \pm 0,22$ & $0,85 \pm 0,57$ & $0,64 \pm 0,18$ & $0,55-0,71$ & 0,07 \\
\hline \multicolumn{7}{|l|}{ Dynamic Balance } \\
\hline VELOC_LL $(\mathrm{em} \%$ s) & $5,10 \pm 1,00$ & $4,70 \pm 0,99$ & $5,00 \pm 0,81$ & $5,00 \pm 0,86$ & $4,3-5,1$ & 0,61 \\
\hline VELOC_AP $(\mathrm{em} \% \mathrm{~s})$ & $2,60 \pm 0,56$ & $2,60 \pm 0,71$ & $2,80 \pm 0,65$ & $2,90 \pm 0,59$ & $0,11-2,3$ & 0,21 \\
\hline CD_LL (em \%) & $81,3 \pm 4,70$ & $80,4 \pm 5,9$ & $81,6 \pm 3,40$ & $80,1 \pm 4,70$ & $0,66-78$ & 0,63 \\
\hline CD_AP $(\mathrm{em} \%)$ & $71,2 \pm 8,5$ & $70,6 \pm 8,6$ & $71,0 \pm 7,30$ & $75,0 \pm 9,40$ & $1,4-67$ & 0,21 \\
\hline
\end{tabular}

NOTE: Values are presented as mean \pm standard deviation. LEGEND: 2TA, pregnant women in the second trimester and active; $2 \mathrm{TS}$, pregnant women in the second trimester and sedentary; 3TA, pregnant women in the third trimester and active; 3TS, pregnant women in the third trimester and sedentary; $95 \%$ CI, 95\% confidence interval; FOA, oscillation speed tested on a firm surface and eyes open; FOF, oscillation speed tested on a firm surface and eyes shut; IOA, oscillation speed tested on an unstable surface and eyes open; IOF, oscillation speed tested on an unstable surface and eyes shut; VELOC LL, speed of oscillation during side-to- side weight shift; VELOC_AP, speed of oscillation during the anteroposterior displacement weight; CD_LL, directional control during side-to-side weight shift; CD_AP, directional control during the anteroposterior displacement weight.
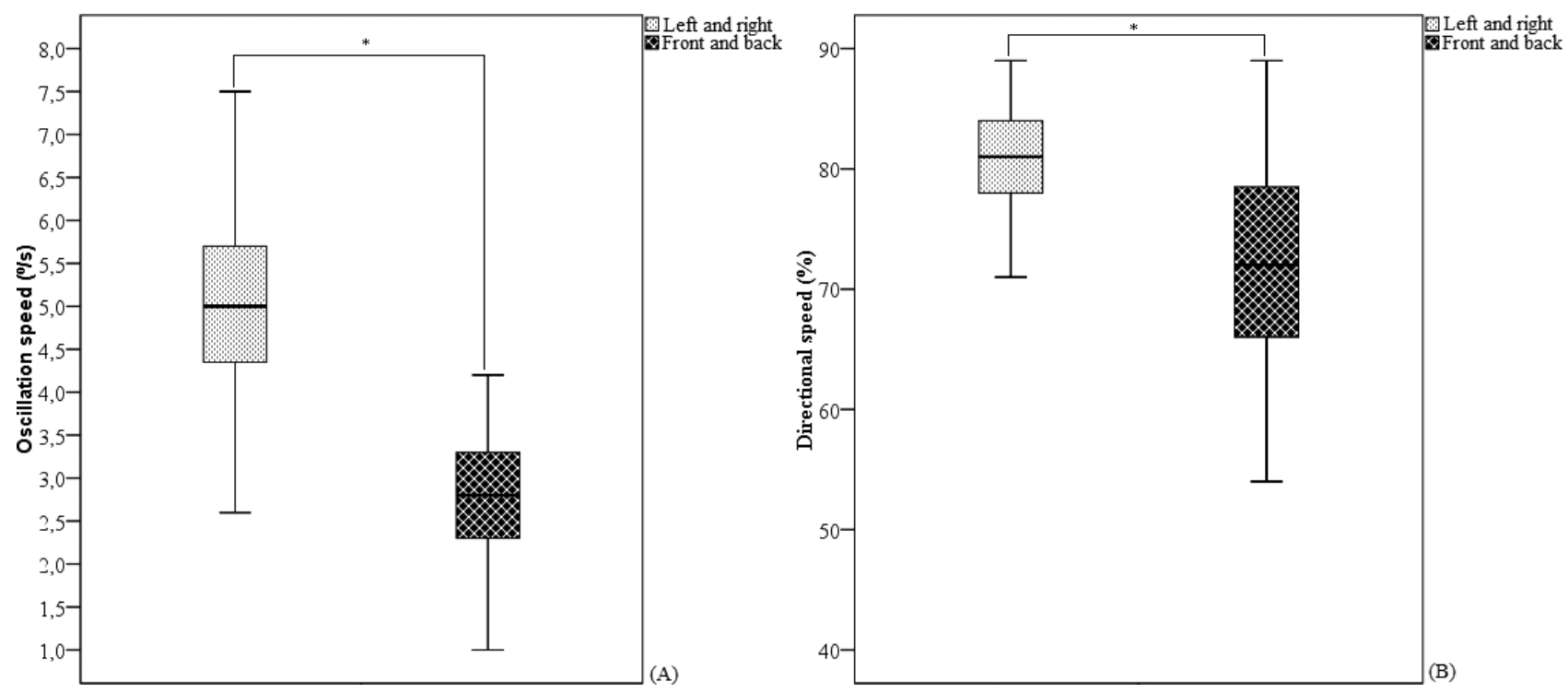

Figure 3 - Analysis if the dynamic postural balance. (A) Comparison of the oscillatory speed during the left/right movements and forward/backward movements. (B) Comparison of directional control during the left/right movements and forward/backward movements. 


\section{Discussion}

\section{Physical Activity and Postural Balance}

The main findings of this study were that physical activity does not influence the static or dynamic postural balance of pregnant women. Possible reasons for this are the following: (1) The musculoskeletal demands required in the clinical setting differed from those evaluated in this study; (2) insufficient frequency of physical activity, and (3) the variability in clinical exercise protocols applied to the pregnant women.

The physical activities most commonly practiced by the volunteers were Pilates (26.1\%), water aerobics (13\%), and walking (13\%). Nascimento, Surita, Cecatti ${ }^{12}$, in a systematic review, found a high frequency of physical activities such as Pilates, yoga, walking, and dancing among pregnant women ${ }^{12}$. Current recommendations targeted to healthcare professionals encourage the incorporation of strength and flexibility exercises into physical activity programs aimed at pregnant women. For this reason, Pilates, water aerobics, and walking are often chosen by women during gestation ${ }^{12}$.

Although these improve postural balance ${ }^{8,10,13}$, there are few studies and physical activity protocols for use in clinical settings that address the different sensory situations of balance training. This could explain the absence of significant differences between the groups. This argument rests on the principle of specificity, based on the fact that motor learning is more effective when training sessions and environmental conditions mimic those required during the execution of the $\operatorname{task}^{14}$. Thus, we can assume that the exercises done by the subjects during this research involved neither a wide repertoire of surface diversity (firm or unstable) nor sensory conditions (eyes open or eyes closed). In addition, it is possible that these activities do not emphasize the dynamic and voluntary displacement of weight in both directions (ante-posterior or side-to-side). This hypothesis seems to be confirmed by the fact that there was a statistically significant difference between the sedentary and active groups only in the speed of oscillation during the static test on a firm surface with eyes open $(\mathrm{P}=0.03)$. This condition is similar to those commonly applied in the clinical setting. Thus, it appears that active pregnant women have more efficient motor learning process, which reflected their superior performance in maintaining balance. A study conducted by Opal-Berdzik and colleagues, who evaluated 31 pregnant and postpartum women (active and sedentary), found similar results ${ }^{1}$.

Another factor that could explain the lack of a relationship between physical activity and postural balance is the frequency of physical activity in pregnant women studied. The American College of Obstetricians and Gynaecologists recommends that pregnant women should practice 30 minutes of moderate activity as many days a week as possible ${ }^{15}$. This recommendation was not followed by the group of volunteers study, given that $41.7 \%$ of them exercised only twice a week. Makara-Studzinska and colleagues, who conducted a survey to analyze the type and frequency of exercises chosen by 100 pregnant women, found similar results, with $37 \%$ of the sample engaged in physical activity only once a week ${ }^{16}$. This suggests that the frequency of physical activity among participants in this research was insufficient for ensuring significant benefits in postural balance among active pregnant women.

Finally, another factor that may explain the lack of a relationship between postural balance and physical activity is the variability in the clinical protocols that active volunteers submitted. As noted, pregnant women participated in many different activities, both in isolation and along with other activities. Some of these activities promote improvement of the cardiorespiratory system, such as walking, water aerobics, and swimming, while others stimulate strength and flexibility, such as Pilates, yoga, and weight lifting ${ }^{17}$. In addition, the intensity and duration of the exercise may have influenced the results. Thereby, we recommend applying protocol standardization that respects the principles of muscle training and motor learning based on scientific findings in clinical settings.

\section{Visual Afference, Type of Surface, and Postural Balance}

In our analysis of static postural balance, we observed that (1) oscillation speed was higher for the unstable surface (regardless of the presence or absence of visual afferent), and (2) when subjects' eyes are closed, the type of surface (firm or unstable) is irrelevant.

The increase in sway velocity on an unstable surface can be explained by the action of integrating somatosensory information from the central nervous system (CNS). On a non-rigid surface, an overload of mechanisms related to the function of the proprioceptive system, mechanoreceptors, and the skin and joint receptors work together to maintain postural balance ${ }^{18}$. The association of this overload of changes in the center of gravity during pregnancy ${ }^{19}$ could explain the participants' difficulty maintaining postural balance and the resulting higher speed of oscillation on unstable surfaces.

The high speed of oscillation during the closed eyes conditions, is due to the physiological mechanisms related to the maintenance of postural balance. Peripheral vision is considered related to the perception of both one's own movement and postural control ${ }^{20}$. Thus, visual cues play an essential role in maintaining static balance ${ }^{20}$.

A study conducted by Butler and colleagues aimed to evaluate the influence of visual information on the balance of pregnant women. These authors conducted a longitudinal study with 12 volunteers and found that the oscillation speed, in the absence of visual afferent, was higher in women in their second and third trimesters of pregnancy, consistent with the results of this research.

The assessment of dynamic postural balance, on the other hand, showed (1) an increase in sway velocity in the laterallateral direction and (2) a reduction in directional control during the anteroposterior displacement.

A study conducted by McCrory and colleagues evaluated variables related to dynamic postural balance in 41 pregnant women and 40 non-pregnant women. It showed that postural sway velocity was higher in the anterior direction, contrary to 
the findings of this research ${ }^{21}$. However, it is noteworthy that the analysis of the oscillation speed in the lateral-lateral direction was not included in the McCrory's work. Similarly, a study conducted by Inanir, Cakmak, Hisim, Demirturk ${ }^{19}$ with110 women ( 80 pregnant and 30 non-pregnant women) found that oscillation speed was higher in the anterior-posterior direction than side-to-side, regardless the gestational trimesters. Future research is needed to elucidate the biomechanical mechanisms underlying the findings of this study ${ }^{22}$.

In contrast, the assumption that directional control would be lower during movement in the anteroposterior direction was confirmed. The voluntary control of movement activates several areas of the cerebral cortex and is related to the purpose of the task. It can be started, stopped, or ended at any time by the person executing the movement ${ }^{23}$. A proper connection between the CNS and the demands of the peripheral system ensures satisfactory control and thus the maintenance of postural balance during voluntary movement. However, pregnant women experience biomechanical and hormonal changes that could hinder this control, particularly with regard to weight displacement in the anteroposterior direction.

The main biomechanical change during pregnancy is a shift in the anterior superior direction of the gravity center, which leads to greater postural instability ${ }^{19}$. Secondly, relaxation of the joints promotes joint laxity, which can interfere with the postural control feedback system ${ }^{24}$. These pregnancy-related body changes can account for the reduction in directional control in the anteroposterior direction found in this research.

\section{Study Limitations}

The main limitations of the study are the absence of a control group (nuligrávidas women) and a specific instrument to measure the volume of physical activity among the participants.

We also note that the findings of this study, which show a lack of a relationship between physical activity and postural balance in pregnant women, should be considered with caution. Another point of interest is the increase in sway velocity under different sensory conditions. These results call attention to the need for physical activity guidelines for clinical use to be based on the principles of muscle training and motor learning. In addition, future studies should incorporate different exercises carried out in different areas and under different sensory conditions. This will enable physiotherapists who work with pregnant women to stimulate the proper conditioning of their postural control systems throughout varied activities, which will improve postural balance and therefore help to reduce the risk of falls among pregnant women.

Therefore, we recommend more open dialogue between academic knowledge and clinical practice with regards to physical activity, diversity of stimuli in the exercises, and postural balance. Future perspectives on the subject should include longitudinal studies on the effectiveness of exercise protocols for pregnant women and the analysis of possible differences in postural balance variables resulting from workout activities.

\section{Conclusion}

The results of this study suggest that (1) physical activity does not influence variables related to postural balance in pregnant women, and (2) postural sway velocity and directional control of movement are related to the test conditions used in the balance assessment.

\section{References}

1. Cieślińska-świder J. Comparison of Static Postural Stability in Exercising and Non-Exercising Women During the Perinatal Period. 2014;1865-70.

2. Pearlman MD, Tintinalli JE, Lorenz RP. A prospective controlled study of outcome after trauma during pregnancy. Am J Obstet Gynecol. 1990;162(6):1502-7; discussion 1507-10.

3. Schiff MA. Pregnancy outcomes following hospitalisation for a fall in Washington State from 1987 to 2004. BJOG. 2008; 115(13):1648-54.

4. Tinker SC, Reefhuis J, Dellinger AM, Jamieson DJ. Epidemiology of maternal injuries during pregnancy in a population-based study. 1997-2005. J Womens Health (Larchmt). 2010;19(12):2211-8.

5. Vladutiu CJ, Evenson KR, Marshall SW. Physical activity and injuries during pregnancy. J Phys Act Health. 2010;7(6):761-9.

6. Swift CG. Care of older people: Falls in late life and their consequences-implementing effective services. BMJ. 2001; 322(7290): 855-7.

7. McCrory JL, Chambers AJ, Daftary A, Redfern MS. Dynamic postural stability in pregnant fallers and non-fallers. BJOG An Int J Obstet Gynaecol. 2010;117(8):954-62.

8. Evenson KR, Moos M-K, Carrier K, Siega-Riz AM. Perceived barriers to physical activity among pregnant women. Matern Child Health J. 2009;13(3):364-75.

9. Gaston A, Cramp A. Exercise during pregnancy: a review of patterns and determinants. J Sci Med Sport. Sports Medicine Australia; 2011; 14(4):299-305.

10. Angyán L, Téczely T, Angyán Z. Factors affecting postural stability of healthy young adults. Acta Physiol Hung. 2007; 94(4): 289-99.

11. Liston R, Brouwer Brenda J. Reliability and Validity of Measures Obtained From Stroke Patients Using the Balance Master. Arch Phys Med Rehabil. 1996;75:425-30.

12. Nascimento SL, Surita FG, Cecatti JG. Physical exercise during pregnancy: a systematic review. Curr Opin Obstet Gynecol. 2012 Dec; 24(6):387-94.

13. Batista DC, Chiara VL. Atividade física e gestação: saúde da gestante não atleta e crescimento fetal Physical activity and pregnancy: non- growth. 2003;3(2):151-8.

14. Corrêa UC. Aprendizagem motora: tendências, perspectivas e aplicações. 2004;55-72.

15. Harrod CS, Chasan-Taber L, Reynolds RM, Fingerlin TE, Glueck $\mathrm{DH}$, Brinton JT, et al. Physical activity in pregnancy and neonatal body composition: the Healthy Start study. Obstet Gynecol. Lippincott Williams and Wilkins; 2014; 124(2 Pt 1):257-64. 
16. Makara-Studzińska M, Kryś-Noszczyk K, Starczyńska M, Sieroń A, Śliwiński Z. Types of physical activity during pregnancy. Polish Ann Med. 2013; 20(1):19-24.

17. Arrais H, Valim-rogatto PC. Influência da especificidade do treinamento resistido sobre aspectos funcionais e antropométricos de homens jovens. Motriz. 2007;288-97.

18. Shaffer, Scott W; Harrison AL. Perspective Aging of the Somatosensory System: A Translational. Phys Ther. 2007;87:193-207.

19. Inanir A, Cakmak B, Hisim Y, Demirturk F. Gait \& Posture Evaluation of postural equilibrium and fall risk during pregnancy. Gait Posture. Elsevier B.V.; 2014;39(4):1122-5.

20. Gaerlan MG, Alpert PT, Cross C, Louis M, Kowalski S. Postural balance in young adults: the role of visual, vestibular and somatosensory systems. J Am Acad Nurse Pract. 2012; 24(6):375-81.

21. McCrory JL, Chambers A, Daftary A, Redfern MS. Dynamic postural stability during advancing pregnancy. J Biomech. Elsevier. 2010.

22. Inanir A, Cakmak B, Hisim Y, Demirturk F. Evaluation of postural equilibrium and fall risk during pregnancy. Gait Posture. Elsevier B.V. 2014; 39(4):1122-5.

23. Mirabella G. Should I stay or should I go? Conceptual underpinnings of goal-directed actions. Front Syst Neurosci. 2014; 8: 1-21.

24. Ersal T, McCrory JL, Sienko KH. Theoretical and experimental indicators of falls during pregnancy as assessed by postural perturbations. Gait Posture. Elsevier B.V. 2014; 39(1):218-23.

\section{Acknowledgments}

We thank the volunteers of this research.

\section{Funding source}

Coordenação de Aperfeiçoamento de Pessoa de Nível Superior (CAPES).

\section{Corresponding author}

Vanessa Patrícia Soares de Sousa

Universidade Federal do Rio Grande do Norte

Email: vanessafisio@gmail.com

Manuscript received on April 24, 2016

Manuscript accepted on July 07, 2016

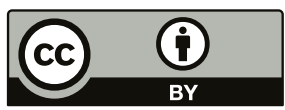

Motriz. The Journal of Physical Education. UNESP. Rio Claro, SP, Brazil - eISSN: 1980-6574 - under a license Creative Commons - Version 3.0 